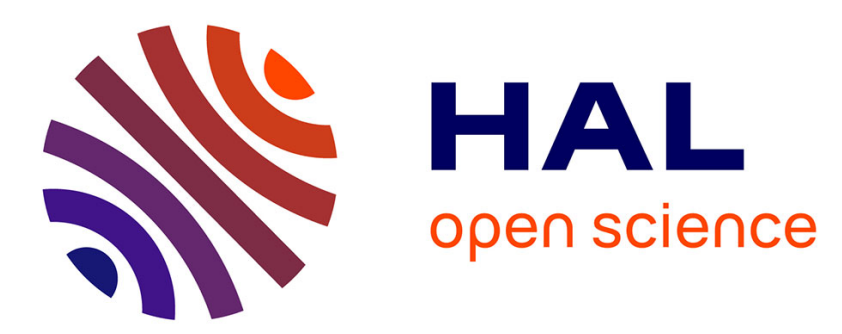

\title{
Les Cecidomyiidae de l'île de la Guadeloupe et récapitulatif des espèces connues des Caraïbes (Diptera)
}

Jean Etienne, Raymond J. Gagné

\section{To cite this version:}

Jean Etienne, Raymond J. Gagné. Les Cecidomyiidae de l'île de la Guadeloupe et récapitulatif des espèces connues des Caraïbes (Diptera). Bulletin de la Société Entomologique de France, 2017, 122 (4), pp.455-466. hal-02622357

\section{HAL Id: hal-02622357 \\ https://hal.inrae.fr/hal-02622357}

Submitted on 26 May 2020

HAL is a multi-disciplinary open access archive for the deposit and dissemination of scientific research documents, whether they are published or not. The documents may come from teaching and research institutions in France or abroad, or from public or private research centers.
L'archive ouverte pluridisciplinaire HAL, est destinée au dépôt et à la diffusion de documents scientifiques de niveau recherche, publiés ou non, émanant des établissements d'enseignement et de recherche français ou étrangers, des laboratoires publics ou privés. 


\title{
Les Cecidomyiidae de l'île de la Guadeloupe et récapitulatif des espèces connues des Caraïbes (Diptera)
}

\author{
par Jean ÉtIENNE ${ }^{1}$ \& Raymond J. GAGNÉ2 \\ ${ }^{1}$ Institut national de la Recherche Agronomique, Centre Antilles-Guyane, Domaine Duclos, \\ F-97170 Petit-Bourg, Guadeloupe <jean.etienne2@wanadoo.fr> \\ ${ }^{2} 606$ St Andrews Lane, Silver Spring, Maryland, 20901, États-Unis <rgagne@rcn.com>
}

(Accepté le 11.X.2017)

Résumé. - Quarante-sept espèces de Cecidomyiidae sont identifiées de Guadeloupe : 33 espèces phytophages dont 14 signalements nouveaux [Asphondylia cordiae Möhn, A. herculesi Möhn, A. lopezae Wünch, A. portulacae Möhn, A. ruelliae Möhn, $A$. stachytarpheta Barnes, $A$. tabernaemontana Möhn, A. waltheriae Möhn, Camptoneuromyia meridionalis Felt, Ctenodactylomyia watsoni Felt, Mycodiplosis rubida (Felt), Neolasioptera portulacae (Cook), Stenodiplosis sorghicola (Coquillett), Youngomyia podophyllae (Felt)]; 1 espèce saprophage; 13 espèces prédatrices dont 7 signalements nouveaux [Arthrocnodax meridionalis Felt, Diadiplosis cocci Felt, D. coccidarum (Cockerell), D. multifila (Felt), D. pulvinariae (Felt), Lestodiplosis fenestra (Felt), L. grassator (Fyles)]. Pour l'ensemble des espèces prédatrices, l'indication de leurs hôtes respectifs est généralement spécifiée. De plus 34 espèces phytophages et 2 espèces prédatrices sont identifiées uniquement au niveau générique et mentionnées. Un tableau indique les 90 espèces de Cecidomyiidae connues actuellement des Caraïbes.

Abstract. - The Cecidomyiidae of Guadeloupe Island and a summary of the known species in the Caribbean (Diptera). Forty-seven species of Cecidomyiidae are known from Guadeloupe: 33 phytophagous species including 14 new records [(Asphondylia cordiae Möhn, A. herculesi Möhn, A. lopezae Wünch, A. portulacae Möhn, A. ruelliae Möhn, A. stachytarpheta Barnes, A. tabernaemontana Möhn, A. waltheriae Möhn, Camptoneuromyia meridionalis Felt, Ctenodactylomyia watsoni Felt, Mycodiplosis rubida (Felt), Neolasioptera portulacae (Cook), Stenodiplosis sorghicola (Coquillett), Youngomyia podophyllae (Felt)]; one is saprophagous; 13 are predatory including 7 new records [Arthrocnodax meridionalis Felt, Diadiplosis cocci Felt, D. coccidarum (Cockerell), D. multifila (Felt), D. pulvinariae (Felt), Lestodiplosis fenestra (Felt), L. grassator (Fyles)]. For all the predatory species, the indication of their respective hosts is generally specified. In addition, 34 phytophagous species and 2 predatory species are identified only at generic level. A table shows the 90 species of Cecidomyiidae known currently in the Caribbean region.

Keywords. - Gall Midges, hosts, faunistics, distribution, West Indies, Neotropical Region.

Au cours des années passées, près de 500 échantillons de cécidomyies phytophages ou prédatrices ont été collectés en Guadeloupe. Au total, environ 80 espèces ont été ainsi récoltées. Certaines de ces collectes ont fait l'objet de publications ou de signalements. Dix-huit espèces ont été signalées (GAGnÉ \& Étienne, 1996, 2006, 2009; GAGNÉ \& JASChHoF, 2014) et cinq espèces nouvelles pour la science ont été décrites (GAGNÉ et al., 1999; GAGNÉ \& ÉtIENNE, 2009, 2015). Au total 23 espèces ont ainsi déjà été répertoriées mais celles-ci ne représentent probablement qu'une faible partie des espèces présentes dans l'île.

Dans la liste ci-après, parmi les 33 espèces phytophages 14 sont nouvellement citées pour la Guadeloupe; par ailleurs, 34 autres ne sont identifiées qu'au niveau générique. Parmi les 15 espèces prédatrices signalées, sept sont nouvelles pour la Guadeloupe alors que deux ne sont identifiées qu'au niveau générique. Pour ces dernières, qui sont des auxiliaires parfois très efficaces, leurs hôtes respectifs ont été identifiés grâce aux inventaires réalisés au préalable notamment sur les Aphididae (Étienne, 2005), les Coccoidea (Matile-Ferrero \& Étienne, 2006; Étienne \& Matile-Ferrero, 2008; Étienne et al., 2014) ou pour la seule espèce de Thrips concernée avec l'article de Michel et al. (2008). La majorité des spécimens ont été déposés à Washington (Washington, DC, National Museum of Natural History, Smithsonian Institution), une autre partie se trouve dans la collection du premier auteur. 


\section{Liste des Cecidomyitdae de Guadeloupe}

Les espèces de Cécidomyies phytophages et prédatrices identifiées sont chacune citées par ordre alphabétique. Seules les espèces identifiées au niveau spécifique sont numérotées. Les genres dans lesquels aucune espèce n'a été identifiée au niveau spécifique sont placés en fin de liste. Pour chaque espèce, la localité, la date et les plantes-hôtes ou arthropodes-hôtes sont indiqués ainsi que le ou les auteurs qui ont déjà cité cette espèce.

\section{Cecidomyinde phytophages}

Pour chaque espèce de Cecidomyiidae de Guadeloupe la localisation des collectes est indiquée ainsi que la ou les plantes-hôtes, en précisant les parties du végétal attaqué. La nomenclature utilisée pour les plantes est celle préconisée dans la flore de FouRnET (2002). Enfin, pour ces mêmes espèces, la répartition géographique et les plantes-hôtes connues sont indiquées d'après GAGNÉ \& JASCHHOF (2014).

1. Anapeza tumida Gagné, 2015. - Guadeloupe : Pointe-Noire, Morne-à-Louis, 10.IV et 14.XII.2011, 22.I et 4.V.2012. Cité de Guadeloupe, tous les spécimens ont été obtenus de galles à la face inférieure des feuilles de Psychotria mapourioides DC. (Rubiaceae) (GAGnÉ \& ÉtIENnE, 2015).

2. Asphondylia cabezasae Möhn, 1959. - Guadeloupe : Les Abymes, 11.VII.1990; Sainte-Rose, plage des Amandiers, 16.I.2007; Deshaies, Cluny, 12.X.2010, tous les spécimens obtenus de fleurs de Crotalaria retusa L. (Fabaceae). Cité du Salvador, Guadeloupe de Crotalaria micronata, C. retusa, Gliricidia sepium, Desmodium nicaraguense (Fabaceae).

3. Asphondylia corbulae Möhn, 1960. - Guadeloupe : Saint-Claude, Bains-Jaunes, 25.III.1993, ex fleurs d'Asteraceae indéterminée et Morne-à-1'Eau, 10.I.2007, ex fleurs d'Eupatorium odoratum L. (Asteraceae). Cité du Salvador, Guadeloupe, Trinidad sur Eupatorium sp., Chromolaena odorata, Fleischmannia microstemon (Asteraceae).

4. Asphondylia cordiae Möhn, 1960. - Guadeloupe : Petit-Canal, Anse-Maurice, 21.VII.2006, ex fleurs de Cordia nesophila I.M. Johnston (Boraginaceae). Cité du Salvador et du Brésil sur Cordia dentata et C. alba. Nouveau pour la Guadeloupe.

5. Asphondylia herculesi Möhn, 1959. - Guadeloupe : Bouillante, Pigeon, route de Dambas, 15.II.2001, 23.I.2007, 5.XII.2008, 5.XII.2010, ex fleurs de Vernonia albicaulis Pers. (Asteraceae). Cité du Salvador sur Vernonia canescens Kunth. Nouveau pour la Guadeloupe.

6. Asphondylia lopezae Wünch, 1979. - Guadeloupe : Vieux-Habitants, Bel-Air, 11.II.2011, ex inflorescences d'Amaranthus sp. (Amaranthaceae). Cité de Colombie : Amaranthus dubius, A. spinosus, Iresine angustifolia (Amaranthaceae). Nouveau pour la Guadeloupe.

7. Asphondylia portulacae Möhn, 1959. - Guadeloupe : Vieux-Habitants, Le Bouchu, 24.XII.1985; Petit-Bourg, Duclos, 7.VI.1990 et Baillif, Saint-Louis, 14.III.2005, ex fleurs Portulaca oleracea L. (Portulacaceae). Cité des États-Unis (Floride), El Salvador, Colombie, Petites Antilles, Bolivie et Argentine, obtenu de Portulaca oleracea (Portulacaceae). Nouveau pour la Guadeloupe.

8. Asphondylia ruelliae Möhn, 1959. - Guadeloupe : Gosier, Bas-du-Fort, 6.VIII.1996; Petit-Canal, anse Maurice, 21.VII.2006, ex gousses de Ruellia tuberosa L. (Acanthaceae). Cité du Salvador sur Ruellia albicaulis. Nouveau pour la Guadeloupe.

9. Asphondylia stachytarpheta Barnes, 1932. - Guadeloupe : Deshaies, Cluny, 10.III.2005 ; Morne-à-l'Eau, Vieux-Bourg, route de Babin, 5.X.2007, 25.VIII.2009 et 26.X.2012, ex fleurs de Stachytarpheta jamaicensis (L.) Vahl (Verbenaceae). Cité de Trinidad sur Stachytarpheta cayennensis. Nouveau pour la Guadeloupe.

10. Asphondylia tabernaemontanae Möhn, 1959. - Guadeloupe : Lamentin, Grosse-Montagne, 17.X.2009, ex fleurs de Tabernaemontana citrifolia L. (Apocynaceae). Cité du Salvador sur Tabernaemontana amygdafolia. Nouveau pour la Guadeloupe.

11. Asphondylia vincenti Felt, 1911 (fig. 1). - Guadeloupe : Deshaies, Grande-Anse, 7.X.2011, ex gousses de Ludwigia octovalvis (Jacq.) Raven (Onagraceae). Cité du Salvador, Guadeloupe et SaintVincent de Ludwigia linifolia, L. suffruticosa, L. octovalvis (Onagraceae).

12. Asphondylia waltheriae Möhn, 1959. - Guadeloupe : Bouillante, Thomas, bord de mer, 5.XII.2010 ; 24.V; 2.IX et 19.X.2011; ex boutons floraux de Waltheria indica L. (Sterculiaceae). Cité du Salvador de Waltheria americana. Nouveau pour la Guadeloupe. 
13. Asphondylia websteri Felt, 1917. - Guadeloupe : Pointe-Noire, route des Mamelles, 6.IX.1990, ex gousses sur Mimosa sp (Mimosaceae). Espèce citée des États-Unis (Californie, Arizona, Oklahoma, Texas), Mexique, Honduras, Guatemala, République Dominicaine, Guadeloupe de Medicago sativa, Cyamopsis tetragonoloba, Mimosa sp., Parkinsonia spp. (Fabaceae); Persea americana (Lauraceae) et Simmondsia chinensis (Simmondsiaceae).

Asphondylia sp1. - Guadeloupe : Petit-Bourg, Duclos, 30.XII.1984 et 20.I.1995, ex fleurs d'Ageratum conyzoides L. (Asteraceae).

Asphondylia sp2. - Guadeloupe : Petit-Bourg, Duclos, 16.I.1985 et 2.III.1989; Lamentin, Ravine-Chaude, 3.III.2005 et 3.V.2005, ex fleurs de Vernonia cinerea (L.) Less. (Asteraceae).

Asphondylia sp3. - Guadeloupe : Morne-à-l'Eau, Vieux-Bourg, 12.III.1985, ex fleurs Erythrina corallodendrum L. (Fabaceae).

Asphondylia sp4. - Guadeloupe : Petit-Bourg, Vernou, 4.X.1990, ex gousse Cassia sp (Caesalpiniaceae). Asphondylia sp5. - Guadeloupe : Saint-Claude, Bains-Jaunes, 25.III.1993 ; Saint-Claude, Beausoleil, 26.I.2007; Pointe-Noire, Morne-à-Louis, 13.II.2007; 3.IV.2011；12.III.2012；10.I.2016, tous les exemplaires ex fleurs de Clibadium erosum (Sw.) DC. (Asteraceae).

Asphondylia sp6. - Guadeloupe : Vieux-Habitants, Le Bouchu, 18.VIII.1993, ex fleurs de Malpighia emarginata DC. (Malpighiaceae).

Asphondylia sp7. - Guadeloupe : Bouillante, Pigeon, route de Dambas, 23.IV.1996, ex galles sur feuilles de Pisonia fragrans Dum.-Cours. (Nyctaginaceae).

Asphondylia sp8. - Guadeloupe : Bouillante, Pigeon, route de Dambas, 14.IX.2000 et 23.X.2000, ex gousses de Chamaecrista glandulosa (L.) Greenevar. swartzii (Wikstr.) Irw. \& Barn (Caesalpiniaceae).

Asphondylia sp9. - Guadeloupe : Saint-François, pointe des Châteaux, 24.VIII.2006, ex fleurs Caesalpinia bonduc (L.) Roxb. (Caesalpiniaceae).

Asphondylia sp10. - Guadeloupe : Lamentin, Ravine-Chaude, 11.I.2007, ex fleurs de Mikania micrantha Kunth (Asteraceae).

Asphondylia sp11. - Guadeloupe : Bouillante, 30.X.2009, 5.XII.2010 et 11.II.2011, ex fleurs Tecoma stans (L.) Juss. (Bignoniaceae).

Asphondylia sp12. - Guadeloupe : Petit-Canal, anse Maurice, 2.XI.2009; Deshaies, Cluny, 28.II.2010; anse Bertrand, Cocando et anse Laborde, 2.XII.2010, tous les spécimens ex fleurs de Tabebuia heterophylla (DC.) Britt. (Bignoniaceae).

Asphondylia sp13. - Guadeloupe : Deshaies, Cluny, 11.XI.2010, ex fleurs d'Aegiphila martinicensis Jacq. (Verbenaceae).

Asphondylia sp14. - Guadeloupe : Petit-Bourg, Duclos, 18.XII.2010, ex fleurs d'Elephantopus mollis Kunth (Asteraceae).

Asphondylia sp15. - Guadeloupe : Vieux-Habitants, Bel-Air, 11.II et 18.III.2011; Vieux-Habitants, Le Bouchu, 22-.I.2011, ex fleurs d'Oxalis barrelieri L. (Oxalidaceae).

14. Camptoneuromyia meridionalis Felt, 1910. - Guadeloupe : Deshaies, Cluny, 21.XI.2010, ex fleurs d'Aegiphila martinicensis Jacq. (Verbenaceae); Petit-Canal, plage de l'anse Maurice, 20.II.2011, ex fleurs de Clerodendrum aculeatum (L.) Schlecht. (Verbenaceae). Les plantes-hôtes sont également nouvelles. Espèce citée de Saint-Vincent et Trinidad sur Ipomoea sp. (Convolvulaceae). Nouveau pour la Guadeloupe.

Camptoneuromyia sp. - Deshaies, Cluny, 12.X.2010, ex fleurs de Crotalaria retusa L. (Fabaceae).

15. Clinodiplosis capsici Gagné, 2000 (fig. 2). - Guadeloupe : Baie-Mahault, 25.II.1986 et 4.I.1996; Lamentin, 29.XI.1990, 7.XI.1992; Lamentin, Ravine-Chaude, 25.IX.1992 et 27.XII.1994; Petit-Bourg, Barbotteau, 9.XI.1992 ; Sainte-Rose, Duportail, 3.II.1993 ; Bouillante, Pigeon, Habitation Dumoulin, 3.IX.1999, tous les spécimens obtenus de galles sur Capsicum frutescens L. (Solanaceae) (GAGNÉ et al., 1999). Cité du Costa Rica, Guadeloupe, Guyane française et Porto Rico sur Capsicum frutescens et C. annuum.

Clinodiplosis sp1. (fig. 7). - Guadeloupe : Petit-Canal, 4.X.2005; Morne-à-1'Eau, Vieux-Bourg, route de Babin, 5.X et 17.XI.2007; 22.VIII.2008; 25.VIII et 19.X.2009, tous les spécimens ex galles en bordure de feuilles de Cordia collococca L. (Boraginaceae).

Clinodiplosis sp2. - Guadeloupe : Deshaies, Cluny, 21.X.2009, ex fleurs d'Ouratea longifolia (Lam.) Engl. (Ochnaceae).

16. Contarinia lycopersici Felt, 1911. - Guadeloupe : Saint-François, ferme Demay, 7.II.1995, ex boutons floraux de Lycopersicon esculentum Mill. var. esculentum (Solanaceae). Cité de Belize, de Guadeloupe à Trinidad, Guyana sur Lycopersicon esculentum (Solanaceae). 


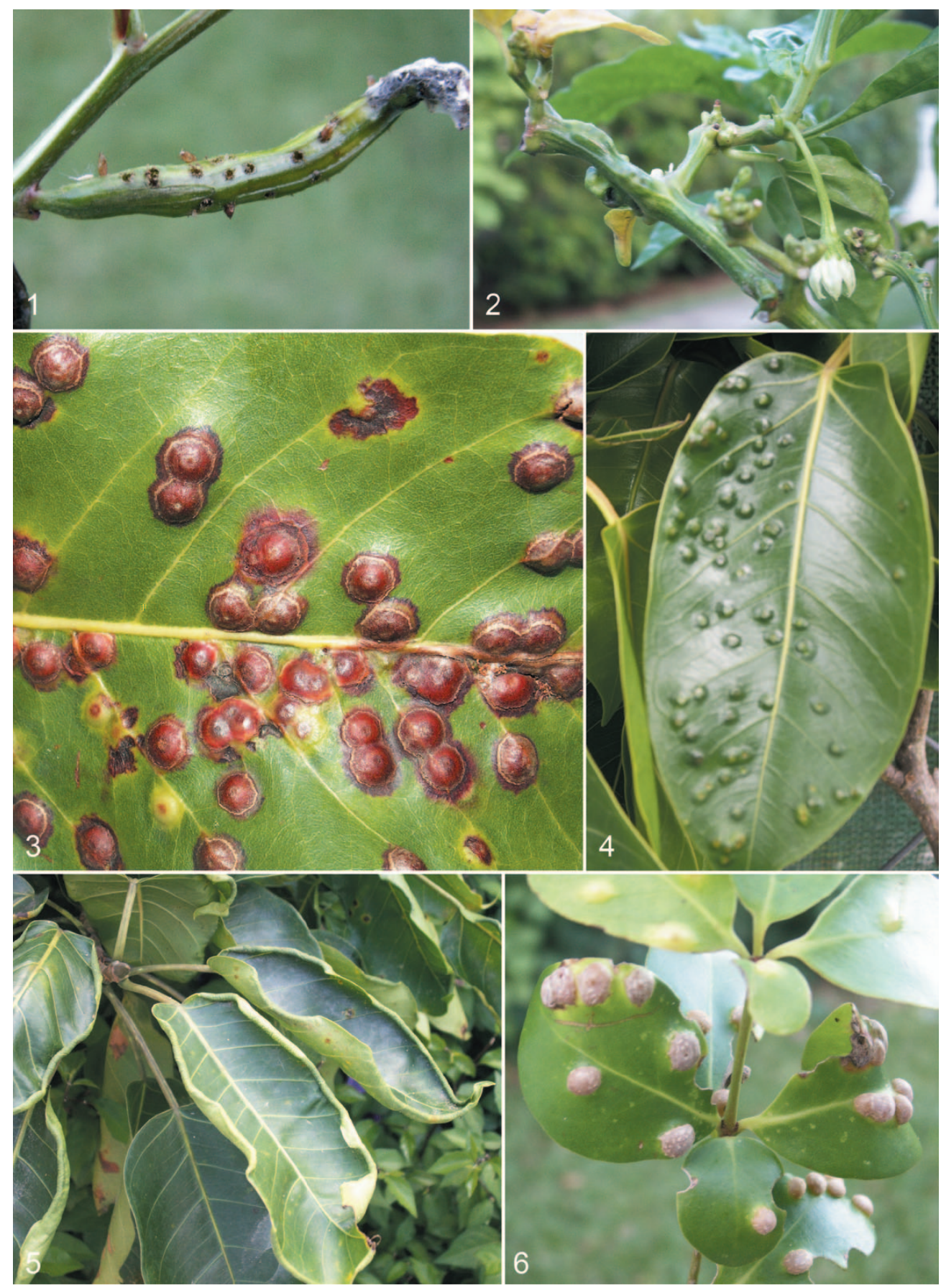

Fig. 1-6. - Galles ou déformations provoquées sur végétaux par des Cecidomyiidae. - 1, Asphondylia vincenti Felt, ex gousses de Ludwigia octovalvis (Onagraceae). - 2, Clinodiplosis capsici Gagné, déformations sur Capsicum frutescens (Solanaceae). - 3, Ctenodactylomyia watsoni Felt, galles sur feuilles de Coccoloba swartzii (Polygonaceae). - 4, Novocalmonia paucula (Gagné \& Étienne), galles sur feuille de Ficus citrifolia (Moraceae). - 5, Autodiplosis sp., galles en bordures de feuilles de Ficus citrifolia (Moraceae). - 6, Bruggmannia sp1, galles sur feuilles de Pisonia fragrans (Nyctaginaceae). 
17. Contarinia maculipennis Felt, 1933. - Guadeloupe : Sainte-Rose, 16.I.1989; Lamentin, Ravine-Chaude, 10.V.1989 et 13.I.1995, ex fleurs d'Hibiscus rosa-sinensis L. (Malvaceae). Petit-Bourg, prise d'eau, 14.VIII.2008, ex fleurs de Dendrobium sp. (Orchidaceae). SERRA et al. (2003) signalent l'introduction de C. maculipennis en 2001 en République Dominicaine et Minatchy et al. (2012) à la Réunion en 2012. Cité de Thaïlande, Vietnam, japon, Hawaii, Guadeloupe, sur Hibiscus sp., Brassica rapa subsp. chinensis (L.) Hanelt (Brassicaceae), Dendrobium (Orchidaceae), Jasminum spp. (Oleaceae), Lycopersicon spp. (Solanaceae), Momordica charantia L. (Cucurbitaceae).

Contarinia sp1. - Guadeloupe : Saint-François, pointe des Châteaux, 31.VIII.2006, ex jeunes pousses et vieilles fleurs de Caesalpinia bonduc (L.) Roxb. (Caesalpiniaceae).

Contarinia sp2. - Guadeloupe : Petit-Canal, Godet, 31.X.2009, ex fleurs de Ziziphus mauritiana Lam. (Rhamnaceae).

Contarinia sp3. - Guadeloupe : Deshaies, Cluny, 21.XI.2010, ex fleurs d'Aegiphila martinicensis Jacq. (Verbenaceae)

18. Ctenodactylomyia watsoni Felt, 1915 (fig. 3). - Guadeloupe : Bouillante, Pigeon, route de Dambas, 15.II.2005, ex galles sur feuilles de Coccoloba venosa L.; 1.XI.2012, 22.I et 15.III.2013 ex galles sur feuilles de Coccoloba swartzii Meissn. (Polygonaceae). Cité des États-Unis (Floride) sur Coccoloba diversifolia, C. uvifera. Nouveau pour la Guadeloupe.

19. Dasineura corollae Gagné, 1977. - Guadeloupe : Saint-Claude, Matouba, Beausoleil, 21.IV.1994, ex fleurs d'Eupatorium dusii Urb. (Asteraceae). Cité de Guadeloupe et Trinidad sur Chromolaena odorata (L.) R. M. King \& H. Rob. et Eupatorium sp.

20. Faramitella planicauda Gagné, 2015. - Guadeloupe : Saint-Claude, Beausoleil, 2.IX.2011, 1.XI.2012; Bouillante, Pigeon, 1.XI.2012, 22.I et 15.III.2013, 17.V.2014, tous les spécimens ex galles sur feuilles de Faramea occidentalis (L.) A. Rich. (Rubiaceae) (GAGNÉ \& ÉtIenNE, 2015).

21. Ficiomyia perarticulata Felt, 1922. - Guadeloupe : Pointe-Noire, Mahault, 11.VII.1995; Petit-Canal, Duval, 30.VII.2007 et Deshaies, Cluny, 12.VIII.2009, tous les spécimens ex fruits de Ficus citrifolia Mill. (Moraceae). Cité des États-Unis (Floride) et Guadeloupe, sur Ficus citrifolia.

22. Gephyraulus mangiferae (Felt, 1927). - Guadeloupe : Vieux-Habitants, Bel-Air, 14.IV.2004; 17.V.2004 ; 9.I.2006; Vieux-Habitants, 17.II.2005 ; Vieux-Habitants, Le Bouchu, 9.I.2006, tous ces spécimens ex fleurs de Mangifera indica L. (Anacardiaceae) (GAGNÉ \& ÉTIENNE, 2006). Cité d'Inde, Hawaii, Porto Rico et Guadeloupe, sur M. indica.

23. Iatrophobia brasiliensis (Rübsaamen, 1908). - Guadeloupe, Petit-Bourg, Duclos, 13.XII.1984; PetitBourg, Duquerry, 29.III.1985; Petit-Bourg, Belle-Vue, 31.V.1985 et Lamentin, Ravine-Chaude, 16.I.1996, tous ces spécimens ex galles sur feuilles de Manihot esculenta Crantz (Euphorbiaceae). Cité du Costa Rica, Guadeloupe, Saint-Vincent, Trinidad, Guyana, Surinam, Brésil, sur Manihot esculenta.

24. Meunieriella avicenniae (Cook, 1909). - Guadeloupe : Goyave, plage Sainte-Claire, 19.XII.1991; 30.I.1992; 20.XII.1994; 17.I et 4.V.1995; 17.V.2006; 6.IV.2011. Sainte-Rose, plage des amandiers, 11.VIII.2009, tous les spécimens ex galles sur feuilles d'Avicennia germinans (L.) L. (Avicenniaceae) (Gagné \& ÉtIenne, 1996). Cité de Cuba, Guadeloupe, États-Unis (Floride), sur A. germinans.

25. Mycodiplosis rubida (Felt, 1911). - Guadeloupe : Lamentin, Ravine-Chaude, 28.I et 25.II.2008, sur feuilles de Commelina diffusa Burm. (Commelinaceae) infestées par les champignons Phytophtora, Colletotrichum, Nigrospora et Arthrobotrys (identification G. Jacqua, Pathologiste INRA-Antilles Guyane). Cité de la Jamaïque, Saint-Vincent, Brésil, Hawaii. Nouveau pour la Guadeloupe.

26. Neolasioptera portulacae (Cook, 1906). - Guadeloupe : Vieux-Habitants, anse la Barque, 2.IX.1993. Baillif, Saint-Louis, 14.III.2005, ex tiges déformées de Portulaca oleracea L. (Portulacaceae). Cité de la région Circumcaribéenne. Nouveau pour la Guadeloupe.

Neolasioptera sp. - Guadeloupe : Bouillante, Pigeon, 31.X.1995, ex feuilles de Passiflora sp. (Passifloraceae).

27. Novocalmonia paucula (Gagné \& Étienne, 2009) (fig. 4). - Guadeloupe : Pointe-Noire, Mahault, 13.II.1995 et 11.VII.1995; Petit-Canal, Duval, 27.VII.2007; Petit-Canal, parc paysager, 18.IV.2008; Deshaies, Cluny, 12.VIII.2009; Petit-Canal, anse Maurice, 28.VIII.2009; tous les spécimens ex galles sur feuilles de Ficus citrifolia Mill. (Moraceae).

28. Pellacara postica Gagné, 2015. - Guadeloupe : Trois-Rivières, Moscou, 20.VII.2000 ; Pointe-Noire, Morne-à-Louis, 10.V.2011, 19.X.2011, 14.XII.2011, 23.III.2012, 22.I.2013 et 11.I.2015, tous les spécimens ex galles sur feuilles de Psychotria mapourioides DC. (Rubiaceae) (GAGNÉ \& ÉTIENNE, 2015). 
29. Procontarinia mangiferae (Felt, 1911). - Guadeloupe : Vieux-Habitants, Bel-Air, 14.II.2000, ex fleurs de Mangifera indica L. (Anacardiaceae). Cité d'Inde, Réunion, Iran, Guadeloupe, Sainte-Lucie, Saint-Vincent, Trinidad, Brésil, sur M. indica.

30. Schizomyia ipomoeae Felt, 1910. - Guadeloupe : Saint-Claude, Matouba, 14.II.1995, ex fleurs d'Ipomoea sp. (Convolvulaceae). Cité de Guadeloupe, Saint-Vincent, sur Ipomoea sp.

31. Schizomyia stachytarphetae Barnes, 1932. - Guadeloupe : Deshaies, Cluny, 8.VII.1993, ex fleurs de Stachytarpheta jamaicensis (L.) Vahl (Verbenaceae). Cité de Guadeloupe et de Trinidad, sur Stachytarpheta cayennensis (Rich.) Vahl, S. jamaicensis.

32. Stenodiplosis sorghicola (Coquillett, 1899). - Guadeloupe : Saint-François, 16.X.1986 et 2.II.1987; Vieux-Habitants, 14.I.1987; Le Moule-Gardel, 2.II et 15.VI.1987, ex panicules de Sorghum bicolor (L.) Moench (Poaceae). Baie-Mahault, Jarry, 1.VI.2010, ex panicules Sorghum arundinaceum (Willd.) R. \& S. (Poaceae). Cité d'Afrique, Inde, Philippines, États-Unis (Sud), Mexique, République Dominicaine, Argentine sur Sorghum bicolor et Sorghum spp. Nouveau pour la Guadeloupe.

33. Youngomyia podophyllae (Felt, 1907). - Guadeloupe : Deshaies, Cluny, 21.XI.2010, ex fleurs d'Aegiphila martinicensis Jacq. (Verbenaceae). Espèce citée des États-Unis (Maine, New York, Pennsylvanie, Virginie, Caroline du Sud), Guatemala, Panama. Nouveau pour la Guadeloupe.

Arcineva sp. - Guadeloupe : Saint-Claude, Beausoleil (alt : 748 m), 17.V.2012, ex fleurs de Faramea occidentalis (L.) A. Rich. (Rubiaceae).

Autodiplosis sp. (fig. 5). - Guadeloupe : Lamentin, Grosse-Montagne, 27.XII.2008; 20.II, 10.VIII, 23.IX, 17.X et 24 .XI.2009, ex galles en bordure de feuilles de Ficus citrifolia Mill. (Moraceae).

Bruggmannia sp1 (grosses galles; fig. 6). - Guadeloupe : Sainte-Rose, Grande-Anse, 4.III.1985; Bouillante, Pigeon, route de Dambas, 23.IV.1996 et 18.VI.1996; Gosier, fort Fleur-d’Épée, 30.IV.1996; Petit-Canal, parc paysager, 18.IV.2008; Anse-Bertrand, pointe de la Grande-Vigie, 1.IV.2011; VieuxHabitants, Bel-Air, 24.V.2011, ex galles sur feuilles de Pisonia fragrans Dum.-Cours. (Nyctaginaceae).

Bruggmannia sp2 (galles plates). - Guadeloupe : Bouillante, Pigeon, route de Dambas, 23.IV.1996, ex galles sur feuilles de Pisonia fragrans Dum.-Cours. (Nyctaginacae).

Ledomyia sp1. - Guadeloupe : Bouillante, Pigeon, route de Dambas, 14.IX.2000, ex gousses de Chamaecrista glandulosa (L.) Greene var. swartzii (Wikstr.) Irw. \& Barn. (Caesalpiniaceae).

Ledomyia sp2. - Guadeloupe : Bouillante, Pigeon, route de Dambas, 15.II.2005, ex baies de Palicourea crocea (Sw.) Schultes (Rubiaceae).

Lopesia sp1. - Guadeloupe : Deshaies, Cluny, 21.X.2009, ex fleurs d'Ouratea longifolia (Lam.) Engl. (Ochnaceae).

Lopesia sp2. (fig. 8). - Guadeloupe : Deshaies, Grande-Anse, 25.XI.2011, larves dans feuilles enroulées de Lonchocarpus punctatus Kunth (Fabaceae).

Prodiplosis sp1. (fig. 10). - Guadeloupe : Gourbeyre, Gros-Morne-Dolé, 11.XI et 17.XI.2009, ex fleurs de Crescentia cujete L. (Bignoniaceae).

Prodiplosis sp2. - Guadeloupe : Deshaies, Cluny, 13.IV.2016, ex boutons floraux de Tabebuia heterophylla (DC.) Britt. (Bignoniaceae).

Resseliella sp. - Guadeloupe : Petit-Bourg, Arnouville, 24.I.2013, ex fleurs de Vernonia cinerea (L.) Less. (Asteraceae).

Rochadiplosis sp. (fig. 9). - Guadeloupe : Goyave, plage de Sainte-Claire, 31.I.1995, 4.V.1995, 21.XI.1995 ; Sainte-Rose, Les Amandiers, 4.IV.1995; Deshaies, Cluny, 14.VI.2002, 26.V.2006, 8.V.2010, 11.XI.2010, 16.III.2011, 16.V.2012, 13.IV.2016, tous les exemplaires ex galles sur feuilles de Dalbergia ecastaphyllum (L.) Taubert (Fabaceae).

\section{CECIDOMYIIDAE SAPROPHAGE}

1. Chrybaneura harrisoni Gagné, 1968.

Hemiptera Monophlebidae hôte : Icerya seychellarum seychellarum (Westwood, 1855) : Lamentin, Ravine-Chaude, 18.IV, 28.VII et 19.XI.2007 ex spécimens morts.

Hemiptera Coccidae hôte : Phalacrococcus howertoni Hodges \& Hodgson, 2010 : Lamentin, RavineChaude, 18.I.2013, ex jeunes stades mycosés sur feuilles de Piper dilatatum Rich. (Piperaceae). Cité du Costa Rica, Panama, Équateur, Guadeloupe, États-Unis (Géorgie) (GAGNÉ \& JASCHHOF, 2014). 


\section{CECIDOMYIIDAE PRÉDATRICES}

1. Arthrocnodax meridionalis Felt, 1912. - Acarina Eriophyidae hôte : Acalitus simplex Flechtmann \& Étienne, 2002, Morne-à-1'Eau, Vieux-Bourg, route de Babin, 30.VII.2007, sur Ruellia tuberosa L. (Acanthaceae). Cet Eriophyidae avait été décrit à l'origine de Marie-Galante (Flechtmann \& Étienne, 2002). Cité des États-Unis (Floride), Saint-Vincent, Trinidad, Pérou (GAGnÉ \& JaschHof, 2014). Nouveau pour la Guadeloupe.

2. Diadiplosis cocci Felt, 1911. - Hemiptera Coccidae hôte : Pseudokermes vitreus (Cockerell, 1894), Lamentin, Ravine-Chaude, 10.III.2010 sur Calliandra surinamensis Benth. (Mimosaceae).

Cité de Cuba, Saint-Vincent (GAGnÉ \& JASChHOF, 2014). Nouveau pour la Guadeloupe.

3. Diadiplosis coccidarum (Cockerell, 1892). - Hemiptera, Pseudococcidae hôtes :

- Dysmicoccus debregeasiae (Green, 1922), Petit-Canal, anse Maurice, 4.VIII.2006, sur Coccoloba uvifera (L.) L. (Polygonaceae) ; Ferrisia virgata (Cockerell, 1893), Saint-François, Les Hauts, 8.XII.1999, sur Poinsettia sp. Vieux-Habitants, 10.V.2005, sur Annona squamosa L. (Annonaceae);
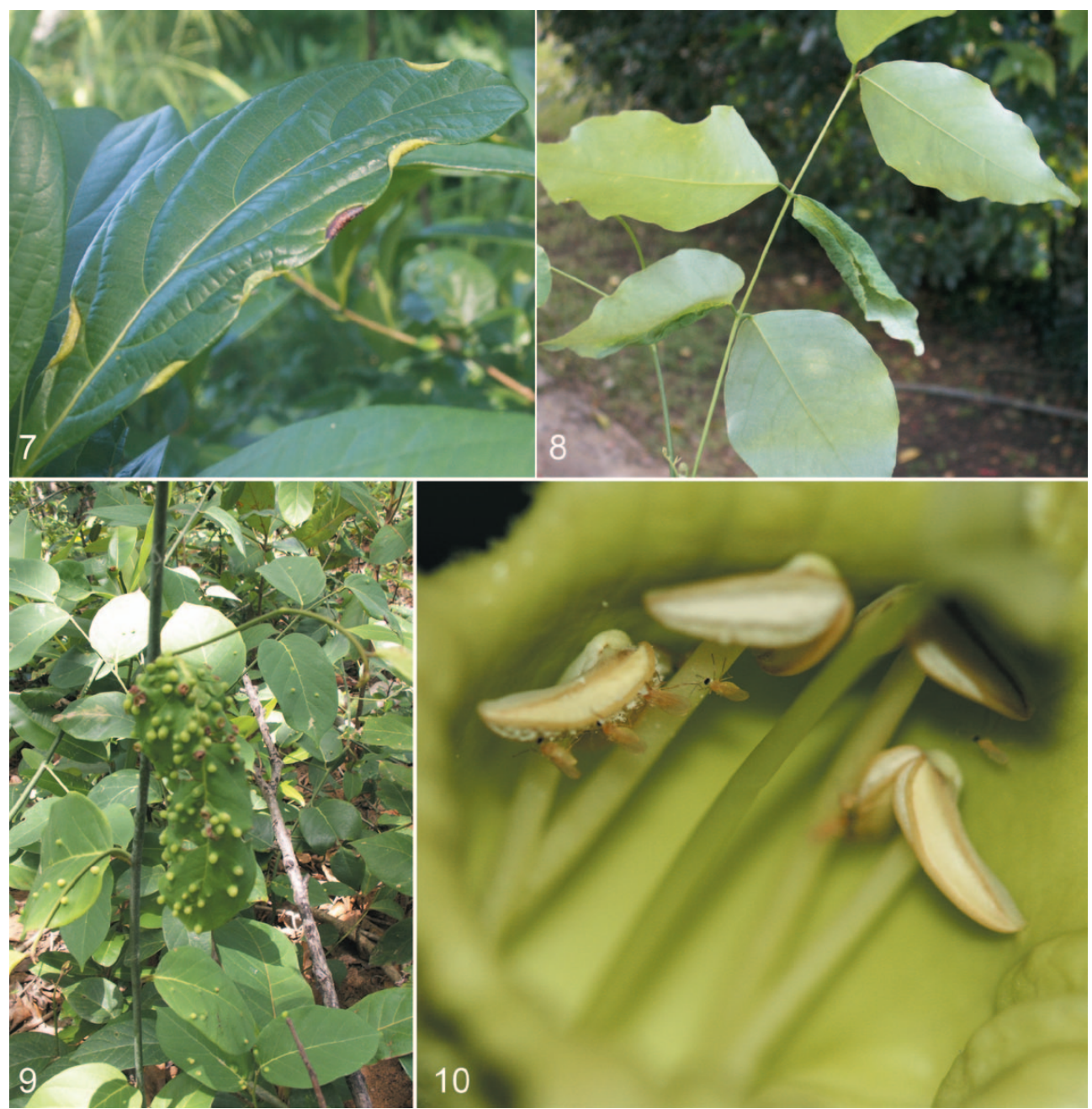

Fig. 7-10. - 7-9, Galles ou déformations provoquées sur végétaux par des Cecidomyiidae : 7, Clinodiplosis sp., galles en bordure de feuilles de Cordia collococca (Boraginaceae); 8 , Lopesia sp., larve dans feuille enroulée de Lonchocarpus punctatus (Fabaceae); 9, Rochadiplosis sp., galles sur feuilles de Dalbergia ecastaphyllum (Fabaceae). - 10, Alimentation d'adultes de Prodiplosis sp. sur pollen dans fleur de Crescentia cujete (Bignoniaceae). 
- Hypogeococcus pungens Granara de Willink, 1981, Lamentin, Ravine-Chaude, 17.II.2000, sur Portulaca quadrifida L. (Portulacaceae);

- Maconellicoccus hirsutus (Green, 1908), Saint-François, 21.V.1999, sur Hibiscus rosa-sinensis L. (Malvaceae);

- Nipaecoccus nipae (Maskell, 1893), Baillif, 26.I.1999, Chrysalidocarpus lutescens Wendl. (Arecaceae);

- Palmicultor palmarum (Ehrhorn, 1916), Baie-Mahault, Fond-Sarail, 4.VI.2006, sur Caryota mitis Lour. (Arecaceae);

- Paracoccus marginatus Williams \& Granara de Willink, 1992, Sainte-Rose, 12.III.1999; PetitBourg, Daubin, 28.VII.1999; Petit-Bourg, Duclos, 28.VI.2000; Baie-Mahault, Belcourt, 17.VII.2000, tous ces échantillons sur Carica papaya L. (Caricaceae). Pointe-Noire, maison du cacao, 17.VIII.2000; Petit-Bourg, Grande-Savane, 31.X.2000, sur Annona muricata L. (Annonaceae). Baie-Mahault, Destrellan, 12.XII.2000, sur Carica papaya (Caricaceae). Saint-Claude, Ducharmoy, 15.III.2001, sur Malvaviscus arboreus Cav. (Malvaceae). Goyave, 5.IV.2001, sur Anacardium occidentale L. (Anacardiaceae). Vieux-habitants, Le Bouchu, 30.III.2001, sur Carica papaya L. (Caricaceae). Petit-Canal, anse Maurice, 18.VIII.2006, sur Pedilanthus tithymaloides (L.) Poit. (Euphorbiaceae);

- Phenacoccus herreni Cox \& Williams, 1981, Petit-Bourg, Duquerry, 25.II.1985 et 29.III.1985; Petit-Bourg, Belle-Vue, 31.V.1985, sur Manihot esculenta Crantz (Euphorbiaceae);

- Phenacoccus madeirensis Green, 1923, Goyave, 13.V.1999, sur Hibiscus rosa-sinensis L. (Malvaceae);

- Planococcus citri (Risso, 1813), Lamentin, Ravine-Chaude, 4.IV.2001, sur Annona muricata L. (Annonaceae);

- Planococcus halli Ezzat \& McConnell, 1956, Petit-Bourg, Duclos, 28.XII.1984, sur Dioscorea sp.

(Dioscoreaceae);

- Spilococcus mamillariae (Bouché, 1844), Pointe-à-Pitre, 24.IV.1987, sur Malpighia emarginata DC.

(Malpighiaceae).

Cité des États-Unis (Floride), de Cuba à Trinidad, Colombie, Guyana, Pérou (GAGNÉ \& JASCHHOF, 2014).

Nouveau pour la Guadeloupe.

4. Diadiplosis coccidivora (Felt, 1911). - Hemiptera Pseudococcidae hôtes :

- Maconellicoccus hirsutus, Vieux-Habitants, Bel-Air, 21.VIII.1998, sur Annona squamosa L. (Annonaceae);

- Paracoccus marginatus, Baie-Mahault, Résidence Muscade, 11.VIII.1998, sur Hibiscus rosasinensis L. (Malvaceae).

Cité des États-Unis (Floride), Bermudes, Jamaïque, Guadeloupe, Panama, Guyana, Brésil, Argentine (GAGNÉ \& JASCHHOF, 2014).

5. Diadiplosis multifila (Felt, 1907). Hemiptera, Pseudococcidae hôtes :

- Planococcus citri, Vieux-Bourg, Lemeslé, 12.III.1985, sur Gossypium hirsutum L. (Malvaceae).

Vieux-Habitants, Bel-Air, 22.IV.1993 et Vieux-Habitants, Le Bouchu, 18.IV.1995, sur Anacardium occidentale L. (Anacardiaceae). Lamentin, Ravine-Chaude, 19.XI.1997, sur Nephelium lappaceum L. (Sapindaceae). Gosier, 30.X.2000, sur Ziziphus mauritiana Lam. (Rhamnaceae);

- Planococcus halli, Le Moule, Gardel, 22.III.1988, sur Cajanus cajan (L.) Huth (Fabaceae);

- Ferrisia virgata, Saint-François, Les Hauts, 8.XII.1999, sur Poinsettia sp. (Euphorbiaceae).

Cité de la République Dominicaine à Trinidad, Brésil et Fidji (GAGNÉ \& JASCHHOF, 2014). Nouveau pour la Guadeloupe.

6. Diadiplosis pulvinariae (Felt, 1912). Hemiptera Coccidae hôtes :

- Philephedra tuberculosa Nakahara \& Gill, 1985, Petit-Bourg, Duclos, Fondor, 22.VI.1992. Baie-

Mahault, Bonardel, 26.V.1995 et Lamentin, Ravine-Chaude, 14.VI.1997, tous sur Gliricidia sepium (Jacq.) Kunth ex Walp. (Fabaceae);

- Pulvinaria urbicola Cockerell, 1893, Petit-Bourg, Duclos, 11.VIII.1994, sur Duranta erecta L. (Verbenaceae).

Cité des États-Unis (Floride), Antilles de la Jamaïque à la République Dominicaine et Trinidad, Venezuela, Guyana (GAGNÉ \& JASCHHOF, 2014). Nouveau pour la Guadeloupe.

7. Diadiplosis vaupedis (Harris, 1968). - Hemiptera, Pseudococcidae hôtes :

- Planococcus halli, Petit-Bourg, Duclos, 13.VI.1997, sur Dioscorea sp. (Dioscoreaceae);

- Planococcus sp., Lamentin, Ravine-Chaude, 3.II.1997, sur Pachystachys lutea Nees (Acanthaceae).

Cité de Colombie, Guadeloupe (GAGNÉ \& JASCHHOF, 2014). 
8. Endaphis maculans (Barnes, 1954). - Hemiptera Aphididae hôte : Aphis gossypii Glover, 1877, PetitBourg, Duclos, 8.I.1985, sur Citrus sp. (Rutaceae).

Cité des États-Unis (Floride), Guadeloupe, Costa Rica, Trinidad, Hawaii (GAGNÉ \& JASCHHOF, 2014). Cité de la région Caraïbe par TANG et al. (1994).

9. Lestodiplosis callipus Gagné, 1977. - Hôte(s) indéterminé(s) : Morne-à-l'Eau, 10.I.2007, ex fleurs d'Eupatorium odoratum L. (Asteraceae); Morne-à-1'Eau, Vieux-Bourg, route de Babin, 17.XI.2007 et 26.X.2012 ex bourgeons et inflorescences de Stachytarpheta jamaicensis (L.) Vahl (Verbenaceae). Cité de Guadeloupe, Trinidad (GAGNÉ \& JASCHHOF, 2014).

10. Lestodiplosis fenestra (Felt, 1908). - Hôte indéterminé : Sainte-Rose, Lamotte, 25.IV.1990, ex fleurs plante indéterminée.

Hemiptera Coccoidea hôtes : Lamentin, Ravine-Chaude, 9.VI.2007, ex Hibiscus rosa-sinensis L. (Malvaceae) infesté par Cerococcus deklei Kosztarab \& Vest, 1966 (Cerococcidae), Maconellicoccus hirsutus (Pseudococcidae), Pinnaspis strachani (Cooley, 1899) (Diaspididae).

Thysanoptera Phlaeothripidae hôte : Holopothrips tabebuia Cabrera \& Segarra, 2008, Petit-Bourg, plage de Viard, 8.I.2009; Lamentin, Bréfort, 13.I.2009, tous les spécimens obtenus des galles de thrips sur feuilles de Tabebuia heterophylla (DC.) Britt. (Bignoniaceae). Ce Thrips décrit en 2008 a envahi la même année toutes les Antilles françaises (Michel et al., 2008).

Cité des États-Unis, New York (GAGNÉ \& JASCHHOF, 2014). Nouveau pour la Guadeloupe.

11. Lestodiplosis grassator (Fyles, 1883). - Hemiptera Coccidae hôte : Ceroplastes ceriferus (Fabricius, 1798), Baie-Mahault, La Jaille, 15.VI.2008 sur inflorescence de Veitchia merrillii (Becc.) H. E. Moore (Arecaceae). Cité de la région néarctique (GAGNÉ \& JASCHHOF, 2014). Nouveau pour la Guadeloupe.

12. Lestodiplosis peruviana Felt, 1911. - Hemiptera, Diaspididae hôte : Diaspis boisduvalii Signoret, 1869, Saint-François, 18.VI.1996, sur Musa sp. (Musaceae).

Cité du Pérou et Guadeloupe (GAGNÉ \& JASCHHOF, 2014).

Lestodiplosis sp. - Hôte indéterminé : Guadeloupe, Lamentin, Grosse-Montagne, 27.XII.2006, 10.VIII et 23.IX.2009, tous les exemplaires obtenus de feuilles de Ficus citrifolia Mill. (Moraceae) avec galles d'Autodiplosis sp.

Lestodiplosis aff. grassator (Fyles, 1883). - Hôte indéterminé : Pointe-Noire, Morne-à-Louis, 3.IV.2011, ex bordures de feuilles enroulées de Myrcia fallax (Rich.) DC. (Myrtaceae) avec présence de larves de Psyllidae et d'Holopothrips sp.

13. Pectinodiplosis erratica Felt, 1908. - Hemiptera Coccidae hôtes :

- Ceroplastes ceriferus, Petit-Bourg, Duclos, 3.VII.2007, sur Mimosa pigra L. (Mimosaceae);

- Parasaissetia nigra (Nietner, 1861), Lamentin, Ravine-Chaude, 17.II.2008, sur Hibiscus rosasinensis L. (Malvaceae).

Hemiptera, Monophlebidae hôte : Crypticerya genistae (Hempel, 1912), Baie-Mahault, Jarry, 14.III et 15.IV.2008, sur Mimosa pigra L. (Mimosaceae).

Cité des Éats-Unis (Washington D.C.), Guadeloupe (GAGNÉ \& JASCHHOF, 2014).

\section{Les Cecidomyitdae de la région Caraïbe}

La répartition géographique dans la région Caraïbe des Cécidomyies connues à ce jour (GAGNÉ \& JASCHHOF, 2014), complétée avec les espèces nouvellement citées dans cette note, est indiquée dans le tableau I. Au total, 90 espèces de Cecidomyiidae ont été répertoriées sur les 13 îles des Caraïbes concernées. Les Grandes Antilles (Cuba, 109884 km²; la Jamaïque, $10991 \mathrm{~km}^{2}$; la République Dominicaine $48442 \mathrm{~km}^{2}$; Porto Rico, $9104 \mathrm{~km}^{2}$ ), qui représentent $95 \%$ de la superficie des 13 îles, ne donnent que $35 \%$ des espèces de Cecidomyiidae identifiées sur l'ensemble des îles. Les 9 autres îles $\left(8941 \mathrm{~km}^{2}\right)$, qui ne représentent que $5 \%$ de l'ensemble de la superficie des îles, fournissent $65 \%$ des espèces connues actuellement. Il apparaît donc clairement que le nombre d'espèces de Cecidomyiidae de cette région est fortement sous-estimé et que la connaissance de ces Diptères est très hétérogène d'une île à l'autre. En effet la Guadeloupe $\left(1434 \mathrm{~km}^{2}\right)$, qui arrive en tête pour le nombre d'espèces identifiées $(47=52,2 \%)$ ne représente même pas $1 \%$ de la superficie totale de l'ensemble des îles $\left(187362 \mathrm{~km}^{2}\right)$. Il en est de même pour Saint-Vincent $\left(345 \mathrm{~km}^{2}\right)$ qui arrive en deuxième position pour le nombre d'espèces recensées $(22=24,4 \%)$ alors que cette île ne représente même pas $0,2 \%$ de la superficie des 
îles concernées. Cuba, la plus grande de toutes les îles avec près de $59 \%$ de la superficie totale des îles prospectées, ne connaît que 15 espèces, soit 16,7 \% des Cecidomyiidae de la région. L'ensemble de ces données ne représente donc pas la réalité mais est seulement le reflet de l'importance des études ou collectes effectuées dans telle ou telle île. Ceci implique qu'un énorme travail de prospection et d'identification reste à réaliser si l'on veut avoir une véritable idée des espèces effectivement présentes dans les Caraïbes.

Tableau I. - Cecidomyiidae des Caraïbes $(\mathrm{Cu}=\mathrm{Cuba} ; \mathrm{Ja}=$ Jamaïque $; \mathrm{RD}=$ République Dominicaine ; $\mathrm{PR}=$ Porto Rico $; \mathrm{IV}=$ Îles Vierges $;$ Mts = Montserrat $;$ Ant $=$ Antigua $; \mathrm{Gu}=$ Guadeloupe $;$ Dom = Dominique ; $\mathrm{StL}=$ Sainte-Lucie $; \mathrm{StV}=$ Saint-Vincent $;$ Bar $=$ Barbade $; \mathrm{Tr}=$ Trinidad $)$.

\begin{tabular}{|c|c|c|c|c|c|c|c|c|c|c|c|c|c|}
\hline Espèces de Cecidomyiidae & $\mathbf{C u}$ & $\mathbf{J a}$ & RD & PR & IV & Mts & Ant & Gu & Dom & StL & StV & Bar & $\operatorname{Tr}$ \\
\hline Anapeza tumida Gagné & & & & & & & & $x$ & & & & & \\
\hline Asynapta citrinae Felt & & & & $x$ & & & & & & & & & \\
\hline Asynapta gosypii (Coquillett) & & & & & & & & & & & & $x$ & \\
\hline Asynapta mangiferae Felt & & & & & & & & & & & & $x$ & \\
\hline Arthrocnodax meridionalis Felt & & & & & & & & $x$ & & & $x$ & & $x$ \\
\hline Asphondylia attenuatata Felt & & & & & & & $x$ & & & & & & \\
\hline Asphondylia boerhaaviae Möhn & & $x$ & & & & & & & & & & & \\
\hline Asphondylia cabezasae Möhn & & & & & & & & $x$ & & & & & \\
\hline Asphondylia corbulae Möhn & & & & & & & & $x$ & & & & & $x$ \\
\hline Asphondylia cordiae Möhn & & & & & & & & $x$ & & & & & \\
\hline Asphondylia herculesi Möhn & & & & & & & & $x$ & & & & & \\
\hline Asphondylia lopezae Wünch & & & & & & & & $x$ & & & & & \\
\hline Asphondylia pattersoni Felt & & & & & & & & & & & $x$ & & \\
\hline Asphondylia portulacae Möhn & & & & & & & $x$ & $x$ & $x$ & $x$ & $x$ & & \\
\hline Asphondylia ruelliae Möhn & & & & & & & & $x$ & & & & & \\
\hline Asphondylia siccae Felt & & $x$ & & & & & & & & & & & \\
\hline Asphondylia stachytarpheta Barnes & & & & & & & & $x$ & & & & & $x$ \\
\hline Asphondylia tabernaemontana Möhn & & & & & & & & $x$ & & & & & \\
\hline Asphondylia vincenti Felt & & & & & & & & $x$ & & & $x$ & & \\
\hline Asphondylia waltheriae Möhn & & & & & & & & $x$ & & & & & \\
\hline Asphondylia websteri Felt & & & & & & & & $x$ & & & & & \\
\hline Atopodiplosis wirthi Gagné & & & & & & & & & $x$ & & & & \\
\hline Bruggmannia pisoniae (Cook) & $x$ & & & & & & & & & & & & \\
\hline Bruggmannia pisonifolia (Felt) & & & & & & & & & & & $x$ & & \\
\hline Bruggmannia pisonioides (Felt) & & & & & & & & & & & $x$ & & \\
\hline Camptoneuromyia meridionalis Felt & & & & & & & & $x$ & & & $x$ & & $x$ \\
\hline Cecidomyia reburrata Gagné & $x$ & & & & & & & & & & & & \\
\hline Chrybaneura harrisoni Gagné & & & & & & & & $x$ & & & & & \\
\hline Clinodiplosis capsici Gagné & & & & & & & & $x$ & & & & & \\
\hline Clinodiplosis cattleyae (Molliard) & & $\times$ & & & & & & & & & & & \\
\hline Clinodiplosis coffeae (Felt) & & & & & & & & & $x$ & & $x$ & & \\
\hline Clinodiplosis eupatorii (Felt) & & & & & & & & & & & $x$ & & $x$ \\
\hline Contarinia gossypii Felt & & & & & $x$ & $x$ & $x$ & & & & & $x$ & \\
\hline Contarinia lycopersici Felt & & & & & & & & $x$ & $x$ & $x$ & $x$ & $x$ & $x$ \\
\hline Contarinia maculipennis Felt & & & $x$ & & & & & $x$ & & & & & \\
\hline Ctenodactylomia coccolobae (Cook) & $x$ & & & & & & & & & & & & \\
\hline Ctenodactylomia watsoni Felt & & & & & & & & $x$ & & & & & \\
\hline Dasineura corollae Gagné & & & & & & & & $x$ & & & & & $x$ \\
\hline Dasineura engeniae Felt & & & & $x$ & & & & & & & & & \\
\hline Diadiplosis cocci Felt & $x$ & & & & & & & $x$ & & & $x$ & & \\
\hline Diadiplosis coccidarum (Felt) & $x$ & $x$ & $x$ & $x$ & $x$ & $x$ & $x$ & $x$ & $x$ & $x$ & $x$ & $x$ & $\times$ \\
\hline
\end{tabular}




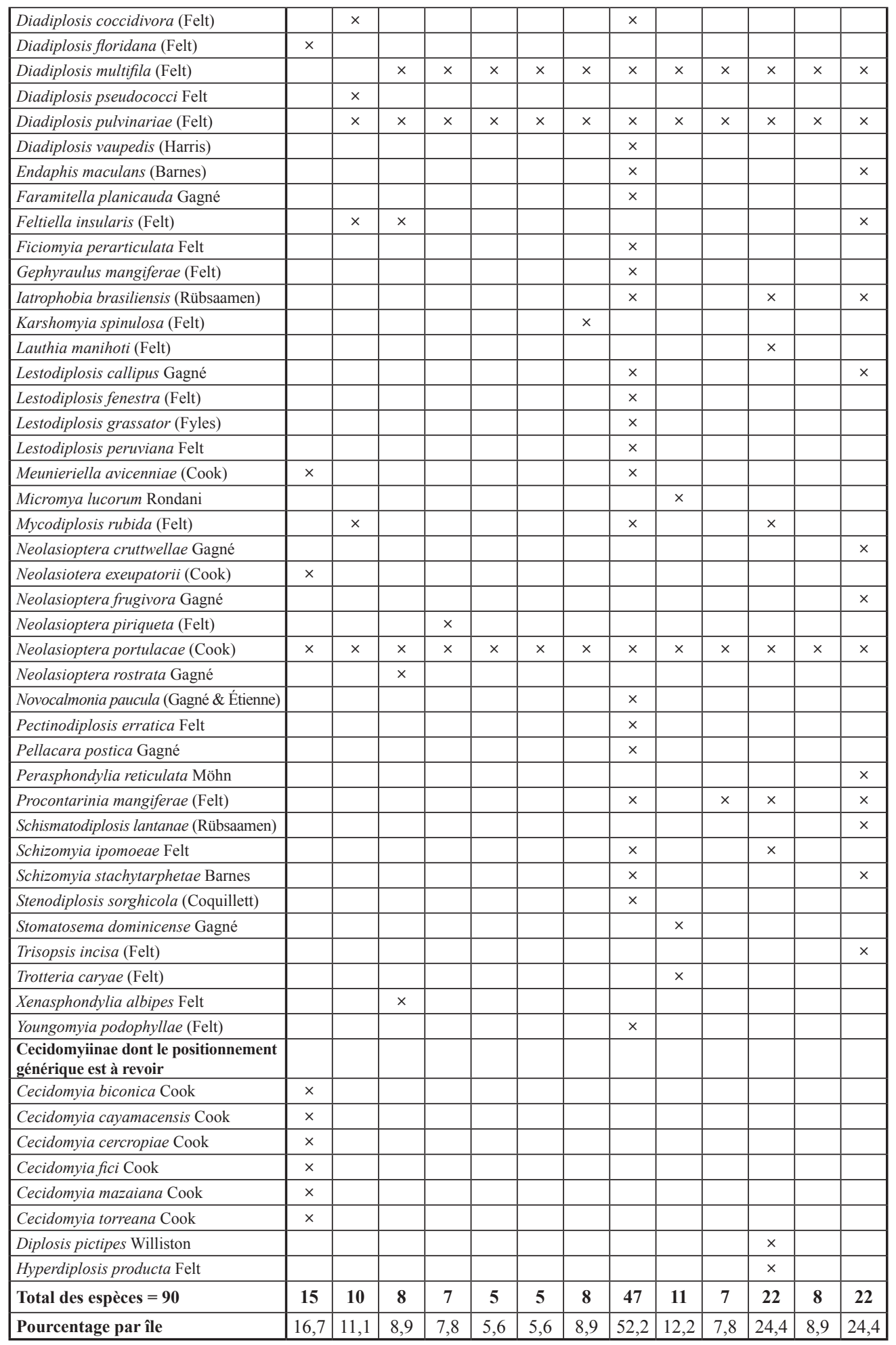


REMERCIEMENTS. - Nous tenons à remercier tout particulièrement notre collègue Danièle Matile-Ferrero (MNHN) qui a permis l'identification de nombreuses cochenilles-hôtes de Cécidomyies. Nos remerciements s'adressent également à notre collègue Daniel Marival (INRA, Antilles Guyane) pour l'aide apportée à la détermination des plantes-hôtes des Cecidomyiidae. Enfin nous tenons à remercier vivement la Direction du Parc National de la Guadeloupe qui nous a autorisés à prospecter dans la zone du parc, permettant ainsi la découverte d'espèces nouvelles.

\section{Auteurs Cités}

Étienne J., 2005. - Les Pucerons de Guadeloupe, des Grandes et Petites Antilles (Hemiptera, Aphididae). Bulletin de la Société entomologique de France, 110 (4/5) : 455-462.

Étienne J. \& Matile-Ferrero D., 2008. - Crypticerya genistae (Hempel), nouveau danger en Guadeloupe (Hemiptera, Coccoidea, Monophlebidae). Bulletin de la Société entomologique de France, 113 (4) : 517-520.

Étienne J., Matile-Ferrero D. \& Kondo T., 2014. - Phalacrococcus howertoni Hodges \& Hodgson (Hemiptera: Coccoidea: Coccidae), a new soft scale record for the Island of Guadeloupe. Revista Corpoica-Ciencia y Tecnologia Agropecuaria, 15 (1) : 115-118.

Flechtmann C. H. W. \& Étienne J., 2002. - New records of plant mites (Acari, Acaridae, Tetranychidae) from Marie Galante with descriptions of five new eriophyid species. Zootaxa, 47 : 1-16.

Fournet J., 2002. - Flore illustrée des phanérogames de Guadeloupe et de Martinique. Nouvelle édition revue et augmentée. Cirad \& Gondwana éditions, 2538 p.

Gagné R. J., Blanco-Metzler H. \& Étienne J., 1999. - A new Neotropical species of Clinodiplosis (Diptera: Cecidomyiidae), an important new pest of cultivated peppers. Proceedings of the Entomological Society of Washington, 102 (4) : 831-837.

Gagné R. J. \& Étienne J., 1996. - Meunieriella avicenniae (Cook) (Diptera: Cecidomyiidae) the leaf gall maker of black mangrove in the American tropics. Proceedings of the Entomological Society of Washington, 98 (3) : 527-532.

— 2006. - Gephyraulus mangiferae (Felt), n. comb. (Diptera : Cecidomyiidae): a mango pest from India newly recorded from the Western Hemisphere. Proceedings of the Entomological Society of Washington, 108 (4) : 930-937.

-2009. - Note on the Cecidomyiidae from Guadeloupe (West Indies) with description of a new species of Paracalmonia (Diptera). Bulletin de la Société entomologique de France, 114 (3) : 337-350.

- 2015. - Three new genera and three new species of Lasiopteridi (Diptera: Cecidomyiidae) on Rubiaceae from Guadeloupe, French West Indies, and a key to genera of Neotropical Lasiopteridi unplaced to tribe. Zootaxa, 4028 (4) : 511-526.

GaGné R. J. \& JASChHof M., 2014. - A Catalog of the Cecidomyiidae (Diptera) of the World. $3^{\text {rd }}$ Edition. Digital version 2. http://www.ars.usda.gov/SP2UserFiles/Place/12454900/Gagne_2014_World_ Cecidomyiidae_Catalog_3rd_Edition.pdf

Matile-Ferrero D. \& Étienne J., 2006. - Cochenilles des Antilles françaises et de quelques autres îles des Caraïbes [Hemiptera, Coccoidea]. Revue française d'Entomologie, (N. S.) 28 (4) : 161-190.

Michel B., Étienne J. \& Ovarbury T., 2008. - A new invasive Thrips in the French Antilles: Holopothrips tabebuia (Thys., Phlaeothripidae). Bulletin de la Société entomologique de France, 113 (4) : 419-420.

Minatchy J., Merion S., Pallas R., Pastou D., Roux E. \& Duffourc V., 2012. - Fiche d'identification de Contarinai maculipennis. Fiche FGDON-RÉUNION. 1 p. http://www.bsv-reunion.fr/wp-content/ uploads/2016/09/fiche-cecydomie-fd.pdf

Serra C. A., Jorge P. E., Abud-Antun A. J., Alvarez P. \& Peguero B., 2003. - Invasive alien species in the Dominican Republic: their impact and strategies to manage introduced pests. Proceedings of the Caribbean Food Crops Society, 39 (1): 102-118.

TANG Y. Q., Yокомi R. K. \& GAgNÉ R. J., 1994. - Life history and description of Endaphis maculans (Diptera: Cecidomyiidae), an endoparasitoid of aphids in Florida and the Caribbean Basin. Annals of the Entomological Society of America, 87 (5) : 523-531. 